\title{
Improved Dynamic Mechanical Properties of Modified PTFE Jet Penetrating Charge with Shell
}

\author{
B. H. Chang, J. P. Yin, ${ }^{1}$ Z. Q. Cui, and T. X. Liu
}

School of Mechatronic Engineering, North University of China, Taiyuan, China

${ }^{1}$ yjp123@nuc.edu.cn

A modified polytetrafluoroethylene (PTFE) was produced by the addition of copper powder to improve the mechanical properties and penetration performance of conventional PTFE. Static compression and split Hopkinson pressure bar test analyses verified the improved mechanical properties of the modified PTFE. Shaped charge structure was designed with by applying modified PTFE to liner material, the formation of modified PTFE jet and the process of jet penetrating shell charge were researched by numerical simulation. As compared to Teflon, results demonstrated that the mechanical properties of the modified PTFE have been significantly improved to achieve greater consistency of jet formation, stronger penetration, broadened pore size, and increased damage performance in the absence of a charge shell explosion.

Keywords: charge with shell, modified PTFE, mechanical properties, shaped charge, jet.

Introduction. Polytetrafluoroethylene (PTFE) [1, 2] is widely used in engineering for its excellent mechanical properties. However, the inadequate penetration capability of formed jet made of low-density liner materials could severely affect the penetration efficiency of shell charges. Therefore, the modification of low-density liner materials is necessary and urgent [3]. The addition of copper powder to conventional PTFE not only improves the density but also achieves comprehensive performance to meet the needs of engineering applications [4]. Rae and Brown [5] has conducted previous research on the performance of PTFE in compression and tension loads. A temperature series was undertaken in tension between -50 and $23^{\circ} \mathrm{C}$ at a strain rate of $800 \mathrm{~s}^{-1}$ for samples of DuPont 7A and 7C Teflon by using a Hopkinson bar. Further high strain rate data on Teflon versus temperature was published by Gray et al. [6] and Walley et al. [7]. Khan and Zhang [8] published some room temperature strain-rate sensitivity data for PTFE between $10^{-4}$ and $1 \mathrm{~s}^{-1}$. Ye et al. [9] have comprehensively researched the mechanical properties of modified PTFE filled with bronze powder of various types, shapes and sizes. The results indicated that PTFE composites with $40 \%$ bronze have better mechanical properties. Gutsev et al. [10] study the effect of silicon dioxide and PTFE additives on NiP coating performance under dry (unlubricated) conditions in unidirectional and reciprocating sliding modes in ball-on-flat configuration. Conte and Igartua [11] took into account several aspects of the tribological behavior of the materials under comparative study. Xie et al. [12] have provided numerical analyses to explore the effects of the addition of copper powder into PTFE composites on thermal conductivity. Sorensen [13] presented a series of experiments of nylon, aluminum, steel, and cased Al/PTFE projectiles striking aluminum armor at $2100 \mathrm{~m} / \mathrm{s}$ to examine defeat mechanisms different than classical high-density penetrators. Although scholars worldwide have researched the general mechanical, friction and loss properties of modified PTFE, the lack of study on the dynamic mechanical properties of PTFE under intense dynamic loading conditions currently prohibits the development of more diversified PTFE applications.

\section{Experiment.}

1.1. Static Mechanical Properties of Modified PTFE. Compressed performance is an important and basic mechanical parameter of modified PTFE composites (made up of 
$38.5 \% \mathrm{Cu}$ powders and $61.5 \%$ PTFE), which reflects the deformation resistance of the material under a compression load. In a compression test, the compression strength of the test piece, a sample cylinder of dimensions, $\varnothing 10 \times 10 \mathrm{~mm}$ refers to the quasi-static change per unit area of a PTFE composite loaded with the coaxial stress in the opposite direction until it yields.

The test procedure and method are as referenced in Test Method of Mechanical Properties of Plastics - General Plastic Compression Performance Test Methods (GB/T1039-1992). The corresponding strain rate range of a compression test at room temperature is $0.003 \sim 0.083 \mathrm{~s}^{-1}$, with a test compression rate range of $2 \sim 50 \mathrm{~mm} / \mathrm{min}$; according to $\dot{\varepsilon}=v / l$, can be calculated. Compression tests examine only the engineering stress $\sigma_{\text {eng }}$ and strain $\varepsilon_{\text {eng }}$ of the materials. With the assumption that the material is incompressible, the relationship between true stress and strain and between engineering stress and engineering strain can be expressed as follows:

$$
\begin{gathered}
\sigma=(1-\varepsilon) \sigma_{\text {eng }}, \\
\varepsilon=-\ln \left(1-\varepsilon_{\text {eng }}\right) .
\end{gathered}
$$

The compression strength $\sigma_{b c}$ refers to the maximum compression response force exerted upon the specimen during compression. A Sansi electronic universal testing machine (UTM6104) in displacement control mode was set to various experimental compression speeds for the tested modified PTFE materials. The compression results with varying compression speeds are shown in Fig. 1.
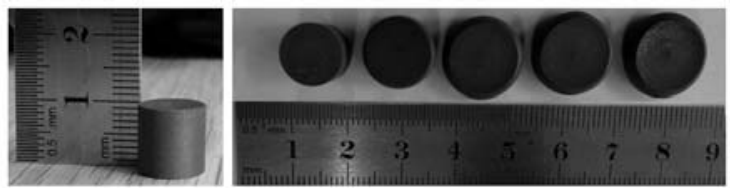

Fig. 1. Compression results of modified PTFE materials under various static conditions.

The compression elastic modulus $369.7 \mathrm{MPa}$ is derived from the linear fitting of straight line segments of the true strain response curves at various compression rates (Fig. 2). In the static compressed hardening stage, the deformation regularity is obtained with different fitting equations: when the power functions displayed in Figs. 2 and 3 fit the data well, results demonstrate high consistency with the experimental curves to obtain $\sigma=A+B \varepsilon^{n}=$ $=15+38 \varepsilon^{0.3253}$ with a correlation coefficient of 0.99694 .

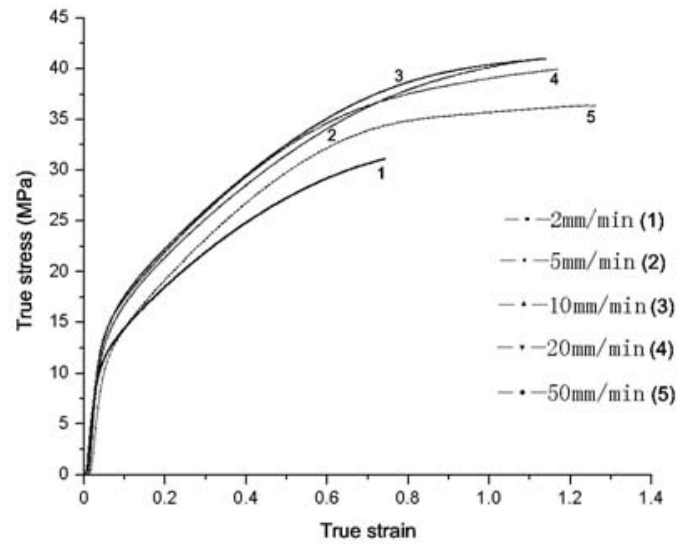

Fig. 2. True strain response curve of modified PTFE.

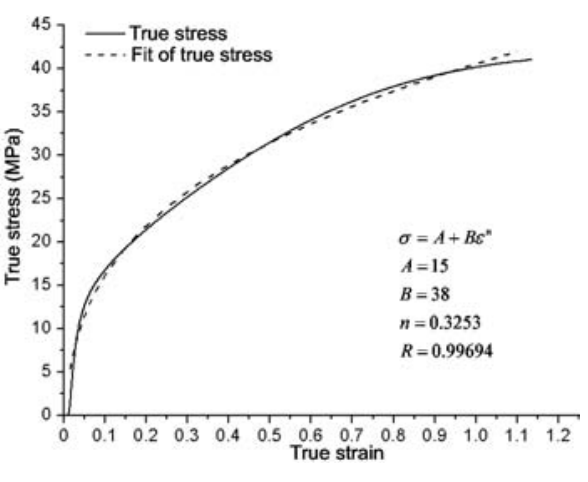

Fig. 3. Curve fitting of modified PTFE. 
1.2. Dynamic Mechanical Properties of Modified PTFE. The Hopkinson experimental system includes an air compressor, air chamber, impact rod, incident bar, transmission rod, buffering rod and energy absorber, rod-positioning support bracket, gas guide tube and a trigger. The data acquisition system contains a speed measuring device of an impact rod which consisting of two parallel laser tubes and two measuring instruments, and the stress wave signal acquisition and recording devices which consisting of the resistance strain gauge, ultra dynamic strain gauge, wheatstone bridge and dynamic data acquisition instrument. The stress-strain relationship of a test piece is indirectly determined by the incident wave $\varepsilon_{i}$ of the strain recorded on the pressing bar, the reflection wave $\varepsilon_{r}$, and the transmission wave signal $\varepsilon_{t}$. Due to the thinness of the test piece, during the impact stress pulse, the stress wave has experienced several round-trip developments in the specimen. The stress rapidly becomes uniform and the test piece reaches stress equilibrium, indicating that the development effects tend to be negligent. The internal strain of short specimens is treated as constant in a uniform state. The internal stress and strain of the test piece is compressed into a plane of zero thickness:

$$
\varepsilon_{i}+\varepsilon_{r}=\varepsilon_{t} .
$$

The response strain rate of the specimens is defined as follows:

$$
\dot{\varepsilon}_{S}=-2 c_{0} \varepsilon_{r} / l_{0} \text {. }
$$

Then, the restrain and stress are defined as

$$
\begin{gathered}
\varepsilon_{s}=-2 c_{0} / l_{0} \int_{0}^{t} \varepsilon_{r} d t \\
\sigma_{s}=E A \varepsilon_{t} / A_{s} .
\end{gathered}
$$

Therefore, the relationship between stress and strain is calculated by the strain gauge of the reflection wave signal and the transmitted wave signal with the impact loading strain rate. A modified PTFE specimen with high strain rate is cylindrical. Split Hopkinson pressure bar (SHPB) compression experiments on various length-diameter ratios of modified PTFE specimens were conducted to determine the fastest optimal balance between the specimen stress wave and the stress of the modified PTFE test specimen to achieve a constant strain rate. Various strain rate impact tests were conducted to determine the dynamic response of the length-diameter ratio under high strain. A series of dynamic compression performance tests of various strain rates on modified PTFE were preceded by SHPB tests of PTFE specimens of various length-diameter ratios. Samples demonstrated plastic strains when the impact velocity of the rod was less than $15 \mathrm{~m} / \mathrm{s}$, samples cracked when the impact velocity further increased to $16 \mathrm{~m} / \mathrm{s}$, the specimens broke into pieces when the impact reached a speed of $18 \mathrm{~m} / \mathrm{s}$ (shown in Fig. 4).
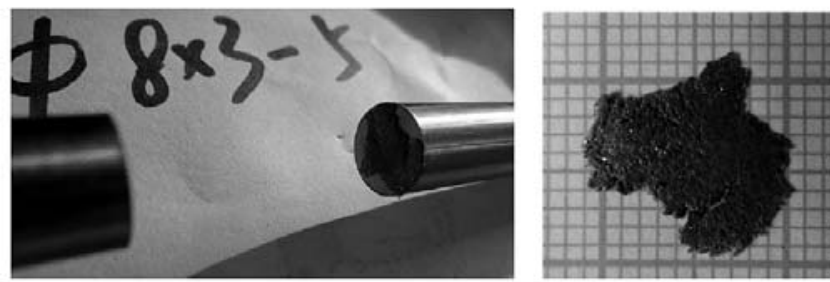

Fig. 4. Breakage of modified PTFE specimen $\varnothing 8 \times 3 \mathrm{~mm}$ with an impact speed of $18 \mathrm{~m} / \mathrm{s}$. 
By setting the pressure parameter on the air compressor to achieve the initial impact on the incident bar and generate a compression stress wave, the strain gauges of the incident and transmission bars were used to monitor stress wave development in the rods. The incident bar recorded the incident and the reflected wave, and the transmission rod recorded the transmission wave. A dynamic strain meter and data acquisition system collected and recorded the generated information. In data processing, MATLAB software of two wave methods was used to reduce the error of signal interference during the test and to process the original data with FFT, so as to achieve a smooth curve, as well as easy image analysis and processing.

Figure 5 depicts a regular recorded pattern of stress wave changes, namely the incident wave, reflection wave and transmission wave during the SHPB test, obtained by the restrain pieces in the incident bar and in the center of the transmission rod. Results indicate a large fluctuation in the initial phase of the incident wave, which is caused by a sharp stress pulse increase at the moment of impact. Fluctuations in the equilibrium position are caused by bar vibration and magnetic interference, as well as other external factors of the environment. The yield strength and maximum strength of modified PTFE under various strain rates are shown in Table 1.

$\mathrm{T}$ a b 1 e 1

Yield and Maximum Strengths of Modified PTFE with Various Strain Rates

\begin{tabular}{||c|c|c|c|c|c||}
\hline Restrain rates $\left(\mathrm{s}^{-1}\right)$ & 1788 & 2280 & 3400 & 4195 & 6195 \\
\hline Yield strength $(\mathrm{MPa})$ & 20.0 & 26.0 & 34.0 & 42.0 & 45.0 \\
\hline Maximum strength $(\mathrm{MPa})$ & 35.0 & 37.5 & 52.5 & 65.0 & 95.0 \\
\hline
\end{tabular}

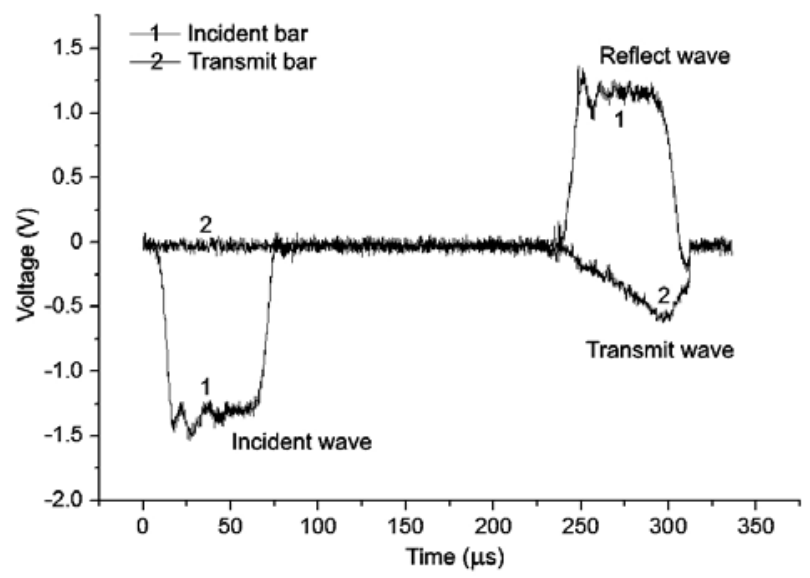

Fig. 5. Elastic wave in the pressing bar.

A high-speed camera was used to record specimen deformation at different impact rates and to analyze the dynamic deformation and failure behavior of the material. The camera operated at a high-speed frequency of $50 \mathrm{MHz}$, one image per $20 \mu \mathrm{s}$, to monitor the axial deformation and observe compression and fracture behavior. Figure 6 displays the impact deformation process at a high-speed image sampling frequency of $50,000 \mathrm{~Hz}$ and nominal sample size $\varnothing 8 \times 3 \mathrm{~mm}$. Compression deformation of the sample begins at the impact site at $20 \mu \mathrm{s}$, swells in the middle at $40 \mu \mathrm{s}$, and then disintegrates in the radial direction at $180 \mu \mathrm{s}$. 


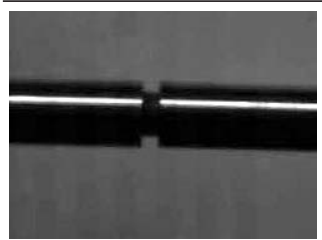

$0 \mu \mathrm{s}$

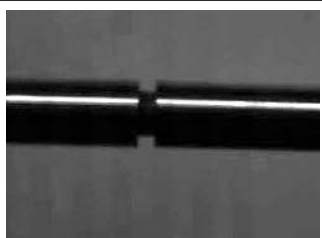

$20 \mu \mathrm{s}$

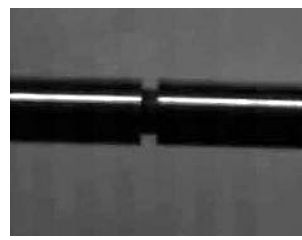

$40 \mu \mathrm{s}$

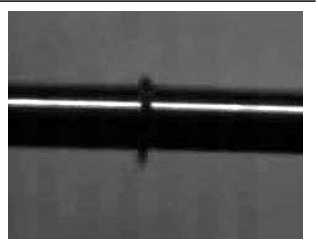

$180 \mu \mathrm{s}$

Fig. 6. Transient deformation process of modified PTFE in $20.8 \mathrm{~m} / \mathrm{s}$.

\section{Numerical Simulation.}

2.1. Model Establishment of Modified PTFE Jet Penetration in Shell Charge. Figure 7 depicts structure of a shaped charge penetration with a fillet processed cone liner, with a cone angle $\alpha=55^{\circ}$, and an average wall thickness and diameter of $40 \mathrm{~mm}$. The height of the explosive charge is approximately equal to the mouth diameter. Without regard for the influence of jet shaping on the shell, a typical 2/4/2 structure is adopted for the shell charge, with a panel thickness of $2 \mathrm{~mm}$, COMPBJJ1B explosive thickness of $4 \mathrm{~mm}$, and a back plate thickness of $2 \mathrm{~mm}$. For practical use of the structure, a $3 \mathrm{~mm}$-thick shield plate was placed in front of the panel. Both shell and shield are made of $30 \mathrm{GrMnSi}$ in a linear state equation, of the von Mises strength model and plastic strain failure model. The Euler algorithm is usually adapted to account for large deformations such as collapse, closeness, jet formation of the liner in an explosion and explosions of a shaped charge.

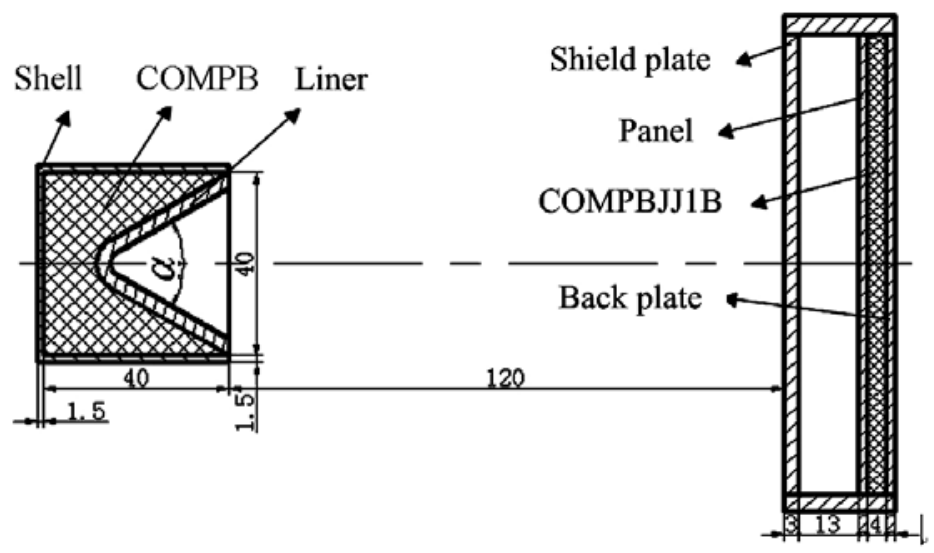

Fig. 7. Shell charge structure of shaped charge penetration.

The specific parameters of a " $\mathrm{B}$ " explosive primarily utilized for numerical simulation calculations are shown as $\rho_{0}==1.717 \mathrm{~g} / \mathrm{cm}^{3}, D=7980 \mathrm{~m} / \mathrm{s}$. The Jones-Wilkins-Lee state equation was used to accurately describe gas pressure, volume and energy characteristic variations in the process of explosion detonation. With COMPBJJ1 shelled charge explosives, the state equation is based on the Lee-Tarver model of ignition and growth, and reflects a good response process upon impact.

Under detonation conditions, the liner material consisting of PTFE and modified PTFE is considered to represent an ideal fluid elastic plastic model, necessitating the use of the von Mises yield and instantaneous destruction criterion and the shock state equation to describe the liner material's dynamic response to the detonation wave. This also allows the calculation of large deformation of the materials subject to a high restrain rate $\left(>10^{5}\right)$. The parameters of two liner materials are shown in Table 2. 


T a b 1 e 2
\begin{tabular}{|c|c|c|c|c|c|c||}
\hline \hline \multirow{2}{*}{ Material } & \multicolumn{7}{c||}{$\begin{array}{c}\text { Parameter } \\
\end{array}$} & $\begin{array}{c}\rho, \\
\mathrm{g} / \mathrm{cm}^{3}\end{array}$ & $\begin{array}{c}G, \\
\mathrm{MPa}\end{array}$ & $\begin{array}{c}\sigma_{y}, \\
\mathrm{MPa}\end{array}$ & $\begin{array}{c}\text { Grüneisen } \\
\text { coefficient }\end{array}$ & $\begin{array}{c}C_{1}, \\
\mathrm{~km} / \mathrm{s}\end{array}$ & $S_{1}$ \\
\cline { 2 - 7 } & 2.16 & 2330 & 50 & 0.9 & 1.34 & 1.93 \\
\hline PTFE & 3.05 & 1370 & 46 & 0.9 & 1.34 & 1.93 \\
\hline Modified PTFE &
\end{tabular}

2.2. Numerical Simulation of the Formation of Modified PTFE Jet. Numerical simulation of the formation of PTFE jet and modified PTFE jet are shown in Figs. 8 and 9. Due to the higher density and lower shear modulus of the modified PTFE, the modified PTFE produces a smaller slug with regular jet in jet tensile process.

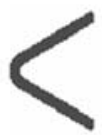

$0 \mu \mathrm{s}$

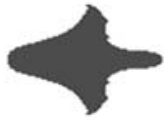

$10 \mu \mathrm{s}$

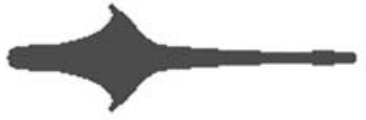

$20 \mu \mathrm{s}$

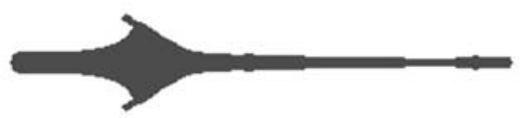

$30 \mu \mathrm{s}$

Fig. 8. Numerical simulation of the formation of PTFE jet at different times.

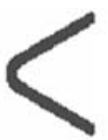

$0 \mu \mathrm{s}$

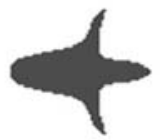

$10 \mu \mathrm{s}$

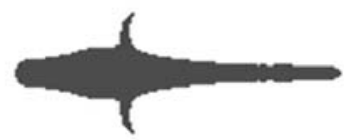

$20 \mu \mathrm{s}$

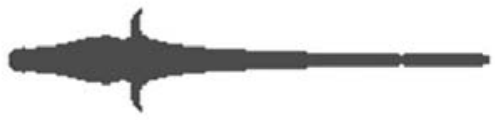

$30 \mu \mathrm{s}$

Fig. 9. Numerical simulation of the formation of modified PTFE jet at different times.

When the detonation pressure is applied to a shaped charge liner, the liner is crushed with the rapid formation of the jet head. Simultaneously, the velocity of a PTFE jet head reaches its maximum of $6278 \mathrm{~m} / \mathrm{s}$ at approximately $8 \mu \mathrm{s}$; the modified PTFE reaches a maximum speed of $5176 \mathrm{~m} / \mathrm{s}$ at $12 \mu \mathrm{s}$. Then the jet stretches and the jet head belongs to slow along a velocity gradient, but a penetrating explosive with a shell still maintains enough speed to achieve effective penetration.

\section{Discussion.}

3.1. Analysis on Degree of Explosive Reaction for Modified PTFE Jet Penetrating Charge with Shell. The impact of the modified PTFE jet on the Shell charge was studied by numerical simulation, and the reaction of the explosive in the shell was obtained by the impact of the jet on the explosive. Figure 10 depicts the high energy of the modified PTFE jet, which leads to a greater degree of explosive reaction without explosion; the energy hinders the jet progress and results in deflagration. Results indicate that the larger jet head diameter of modified PTFE produces greater jet consistency with a larger diameter of perforation from the molding.

\subsection{Damage Properties Analysis on Modified PTFE Jet Penetrating Charge with}

Shell. Jet penetration on the reactive armor shielding plate, panel and back plate can be seen the rod projectile penetration with a certain length and diameter ratio, determining the jet ability to penetrate each baffle layer (shown in Figs. 11 and 12). Based on applied PTFE and modified PTFE to the liner material, the process of jet formation and shell charge penetration were analyzed by ANSYS/LS-DYNA finite software. The results show that the 


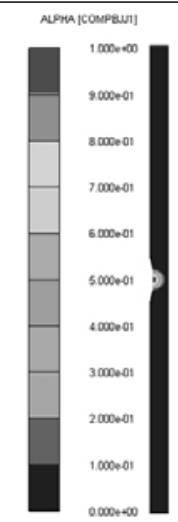

$36 \mu \mathrm{s}$

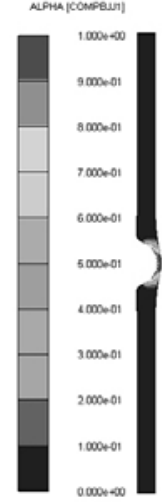

$39 \mu \mathrm{s}$

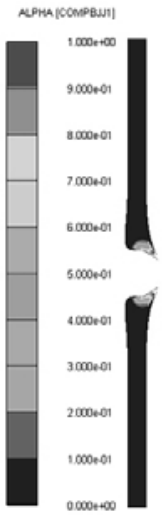

$42 \mu \mathrm{s}$

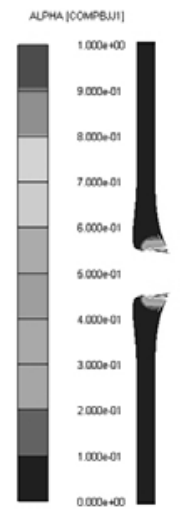

$45 \mu \mathrm{s}$

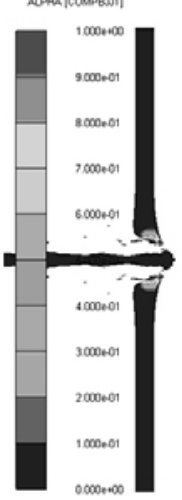

$48 \mu \mathrm{s}$

Fig. 10. Degree of explosive reaction of shell charge impact of modified PTFE.

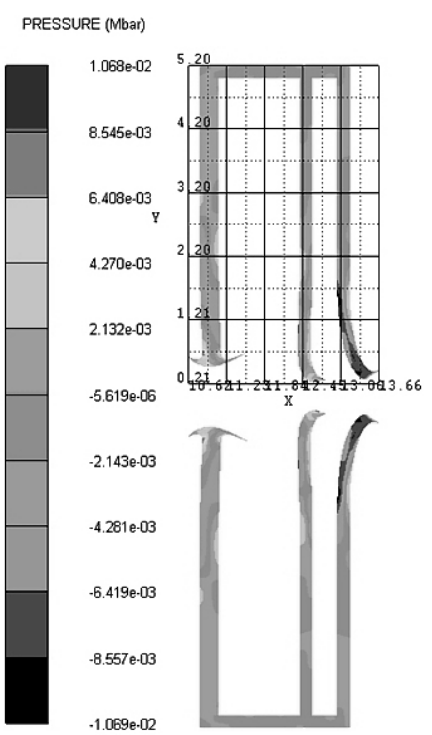

Fig. 11. Aperture of shell by PTFE jet penetration.

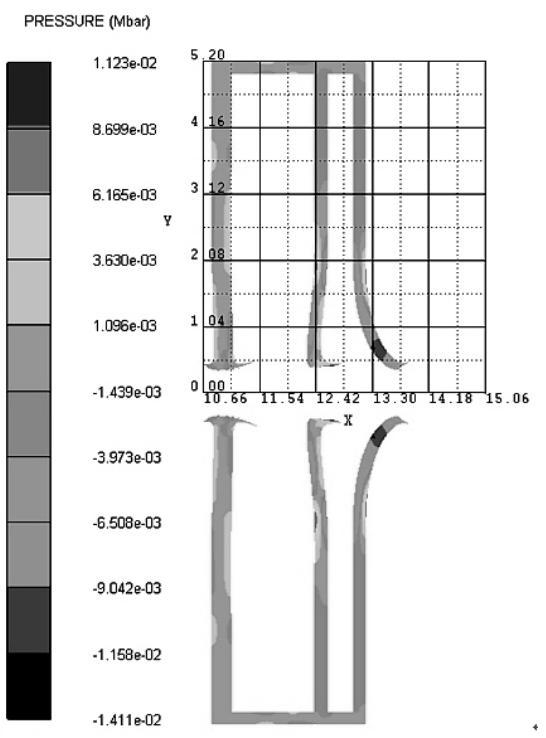

Fig. 12. Aperture of shell by modified PTFE jet penetration.

optimized shaped charge structure is achieved when liner diameter of $40 \mathrm{~mm}$, thickness is $3 \mathrm{~mm}$ and vertex angel is $55^{\circ}$, the jet head speeds up to $4700 \mathrm{~m} / \mathrm{s}$ with a jet diameter of $2.7 \mathrm{~mm}$ at present.

Numerical simulation shows that when PTFE jet penetrates the shell charge, the head diameter is $3.3 \mathrm{~mm}$. When the penetration process ends, the diameter of the shield plate is $4.87 \cdot 2=9.74 \mathrm{~mm}$, the diameter of the panel is $2.52 \cdot 2=5.04 \mathrm{~mm}$, and the diameter of the back plate is $3.1 \cdot 2=6.2 \mathrm{~mm}$. By contrast, when PTFE/Cu jet penetrates the shell charge, the head diameter is $2.7 \mathrm{~mm}$. When the penetration process ends, the diameter of the shield plate is $3.75 \cdot 2=7.5 \mathrm{~mm}$, the hole diameter of the panel is $4.3 \cdot 2=8.6 \mathrm{~mm}$, and the diameter of the back plate is $4 \cdot 2=8 \mathrm{~mm}$. Because PTFE/Cu jet has a higher level of energy than PTFE (so is associated head diameter), the perforation diameter of the panel for $\mathrm{PTFE} / \mathrm{Cu}$ jet is $70.6 \%$ higher than that of PTFE jet; the perforation diameter of the back plate for PTFE/Cu jet is $29.0 \%$ higher than that of PTFE jet. 
Conclusions. The paper has been conducted regarding the static and dynamic mechanical properties of modified PTFE which is made up of $38.5 \% \mathrm{Cu}$ powders and $61.5 \%$ PTFE under dynamic loading conditions. Modified PTFE material was applied to the shaped charge liner structure, Numerical simulation demonstrated that the jet of the modified PTFE forms a larger jet diameter with a greater energy of penetration charge than conventional PTFE.

1. Elastic modulus of modified PTFE under static compression reaches $369.7 \mathrm{MPa}$, and the material tends to be soft and tough. Under static compression, modified PTFE is pressed into a pie. Under dynamic impact, the dynamic yield and maximum strengths of modified PTFE increase gradually, high-speed photographic observations demonstrated high velocity impact breaking the sample into pieces.

2. When the vertex angle of the liner reaches $55^{\circ}$ with thickness of $3 \mathrm{~mm}$, stable jet of modified PTFE penetration is formed with the shell charge. The jet head speeds up to $4700 \mathrm{~m} / \mathrm{s}$ with diameter of $2.7 \mathrm{~mm}$.

3. When PTFE jet and modified PTFE jet penetrating shell charge, the perforation diameter of the panel for modified PTFE jet is 70.6\% higher than that of PTFE jet; the perforation diameter of the back plate for modified PTFE jet is $29.0 \%$ higher than that of PTFE jet, the modified PTFE forms a steady jet to shell charge, which does not ignite the loading explosives inside the case.

Acknowledgments. The authors would like to acknowledge the financial support from Project supported by the National Natural Science Foundation of China under Grant No. 11572291.

1. P. J. Rae and D. M. Dattelbaum, "The properties of poly(tetrafluoroethylene) (PTFE) in compression," Polymer, 45, Issue 22, 7615-7625 (2004).

2. M. Silberberg and R. Supnik, "Tetrafluoroethyene polymers,” in: D. I. McCane (Ed.), Encyclopedia of Polymer Science and Technology, Vol. 13, Wiley, New York (1970), pp. 623-654.

3. F. D. Dong, Z. J. Wang, J. P. Yin, et al., "Numerical simulation on initiating value of low density jet impacting explosive with shell," Ordnance Mater. Sci. Eng., Issue 4, 53-55 (2013).

4. H. L. Li, L. N. Zhu, and S. J. Xie, "Modified polytetrafluoroethylene and its application," Hydraul. Pneum. Seals, No. 6, pp. 4-8 (2012).

5. P. J. Rae and E. N. Brown. "The properties of poly(tetrafluoroethylene) (PTFE) in tension," Polymer, 46, Issue 19, 8128-8140 (2005).

6. G. T. Gray III, C. M. Cady, and W. R. Blumenthal, "Influence of temperature and strain rate on the constitutive behavior of Teflon and nylon," in: A. S. Khan (Ed.), Constitutive and Damage Modeling of Inelastic Deformation and Phase Transformation: Proceedings of PLASTICITY'99: The Seventh International Symposium on Plasticity and its Current Applications, NEAT Press, Fulton, MD (1998), pp. 955-958.

7. S. M. Walley, J. E. Field, and N. A. Safford, "A comparison of the high strain rate behaviour in compression of polymers at $300 \mathrm{~K}$ and $100 \mathrm{~K}, " J$. Phys. IV France, 01 (C3), C3-185-C3-190 (1991).

8. A. Khan and H. Zhang, "Finite deformation of a polymer: experiments and modeling," Int. J. Plast., 17, 1167-1188 (2001).

9. S. J. Ye, Q. Fan, L.Y. Deng, and Y. H. Wang, "The mechanical and tribological properties of copper filled PTFE composites," Lubr. Eng., 35, Issue 9, 60-64, 68 (2010). 
10. D. Gutsev, M. Antonov, I. Hussainova, and A. Y. Grigoriev, "Effect of $\mathrm{SiO} 2$ and PTFE additives on dry sliding of NiP electroless coating," Tribol. Int., 65, 295-302 (2013).

11. M. Conte and A. Igartua, "Study of PTFE composites tribological behavior," Wear, 296, Issues 1-2, 568-574 (2012).

12. T. Xie, Z. J. Lin, G. Chen, et al. "Numerical analysis of influence of $\mathrm{Cu}$ particle content on thermal conductivity of PTFE-based composites," Metal. Funct. Mater., 17, Issue 2, 52-56 (2010).

13. B. Sorensen, "High-velocity impact of encased A1/PTFE projectiles on structural aluminum armor," Proc. Eng., 103, 569-576 (2015). 